\title{
Myelopathy caused by Ossification of Thoracic Ligamentum Flavum
}

\author{
F Yudoyono ${ }^{1,2}$, RH Dahlan ${ }^{1,2}$, SE Ompusunggu ${ }^{1,2}$, L Hamijoyo², MZ Arifin ${ }^{3}$
}

${ }^{1}$ Spine Research Laboratory, Division of Neurospine, Department of Neurosurgery; Faculty of Medicine Universitas Padjadjaran Dr. Hasan Sadikin General Hospital, Bandung, Indonesia

${ }^{2}$ Division of Rheumatology, Department of Internal Medicine, Faculty of Medicine Universitas Padjadjaran Dr. Hasan Sadikin General Hospital, Bandung, Indonesia

${ }^{3}$ Department of Neurosurgery, Faculty of Medicine Universitas Padjadjaran Dr. Hasan Sadikin General Hospital, Bandung, Indonesia

\begin{abstract}
Hypertrophy of the posterior spinal elements leading to compromise of the spinal canal and its neural elements is a well-recognized pathological entity affecting the lumbar or cervical spine. Such stenosis of the thoracic spine in the absence of a generalized rheumatological, metabolic, or orthopedic disorder, or a history of trauma is generally considered to be rare. Spinal ligaments, such as the ligamentum flavum (LF), are prone to degeneration and can lead to back pain and nerve dysfunction. Ossification of ligamentum flavum (OLF) is a pathological condition that cause neurological symptoms and usually occurs in the thoracic spine and less frequently in the cervical spine. However the disease is now being increasingly recognized as a cause of thoracic myelopathy. We report a rare case of thoracic myelopathy caused by OLF. A 48-year-old male presented with a chief complaint of weakness of bilateral lower extremities. Neurological examination revealed sensory deficit at Th 11 level below. Magnetic resonance imaging and computed tomography demonstrated OLF at the right T9-11 level. Thoracic myelopathy caused by OLF was consider and surgical intervention was performed. Posterior decompression and laminoplasty has been performed for this patient. Key words: ossification of ligamentum flavum, thoracic myelopathy, laminoplasty
\end{abstract}

\section{Introduction}

Hypertrophy of the posterior spinal elements leading to compromise of the spinal canal and its neural elements is a well-recognized pathological entity affecting the lumbar or cervical spine. Such stenosis of the thoracic spine in the absence of a generalized rheumatological, metabolic, or orthopedic disorder, or a history of trauma is generally considered to be rare. OLF is the most common compressive factor contributing to thoracic myelopathy in japanese, and was responsible for $60 \%$ of all thoracic cord compression either alone or combination with posterior longitudinal ligament ossification (OPLL) or in East Asia with an incidence rate between $2 \%$ to $4 \%$, Males is predominance $2.2: 1$, and the highest prevalence was in their sixties followed by fifties and seventies ${ }^{1,2}$

Ossification of the ligamentum flavum (OLF) of the thoracic spine is a pathological condition that causes neurological deficit usually occurs in the thoracic spine and less frequently in the cervical spine. ${ }^{4}$ However the disease is now being increasingly recognized as a cause of thoracic myelopathy. ${ }^{5,6}$ Mechanical stress, growth factors and trauma of the spine contribute to accelerated ossification. OLF has been widely recognized as a main cause of thoracic myelopathy in Japanese patients. Few series are reported from Korea, China, India, Middle East, and Caribbean with sporadic case reports in Caucasians. It commonly involves lower thoracic spine (T9-T12) with upper thoracic spine (T1-T4) being the next common site. ${ }^{3,7-9}$ Herewith, we report case of OLF in asian people that were treated in our hospital. The LF is an unusual elastic ligament compared to other spine ligaments because its high composition of elastic fibers. It functions as a passive stabilizing ligament, restoring the spine to a neutral posture following flexion and extension. This spinal ligament is high risk to calcification, ossification, and degenerative hypertrophy changes and the resultant thickening of the tissue has been implicated as a significant cause of stenosis. The LF is composed of elastin and collagen, approximately in a 2:1 ratio in humans. In several mammals the LF has been found to have an elastin content of $40-60 \%$, including humans. This compares to approximately $1 \%$ in skin, 28 $32 \%$ in the aorta, $3-7 \%$ in the lung, and $4 \%$ in the Achilles tendon. In destructive mechanical testing, a lacerated LF reduced the spine's resistance to flexion by $25 \%$, In vitro studies revealed that the rupture strain for the LF averaged nearly $50 \%$, as compared to $4-10 \%$ in the Achilles tendon, and $28 \%$ in the anterior cruciate ligament (ACL). ${ }^{10,11}$

Liang ren et al in recent studies suggested that genetic modifications on numerous growth factors including BMP and TGF- $\beta$ revealed their roles in regulating the development, growth, maintenance as well as the formation of new cartilage and bone tissues, Mobbs had demonstrated dietary habits may also be another independent risk factor. A patient with a low risk genetic background developed multilevel OLF as he followed standard Japanese dietary consumption of pickles (salted products) since young. Secondary to aging process, mechanical stress, disturbance in glucose metabolism, obesity, higher prevalence of diabetes melitus and hyperinsulinism were observed in patients with OLF., 
The mechanism of ligament ossification is classified into five types: Type I, laterally at the origin of the ligamentum flavum at the articular processes; Type II, from the lateral origin of the ligamentum flavum to the interlaminar portion of the ligamentum flavum; Type III, protrudes into the canal posterolaterally but is not fused in the midline; Type IV, consists of bilateral ossified ligaments that are fused at the midline with a groove at the fusion in midline; and Type V, the tuberous type, occurs when the fused ossified ligamentum flavum forms a "tuberous" mass posteriorly in the midline, which protrudes into the spinal canal. According to this classification only types IV and V would develop a myelopathy, and most of types I, II, and III would remain asymptomatic. ${ }^{12-14}$

\section{Case Report}

A 48-year-old male presented in our spine outpatient clinic complains of weakness of bilateral inferior extremities that gradually worsened over a period of ten years. On admission his muscle weakness $3 / 5$ and hypaesthesia below the dermatome Th 11 was revealed. The reflexes were hyperactive in the lower extremities. Deep tendon reflexes were increased at and below the bilateral quadriceps femoris with myoclonus bilaterally. There was no urinary or bowel dysfunction. Plain $\mathrm{x}$-rays of the thoracic spine failed to demonstrate any specific abnormality.

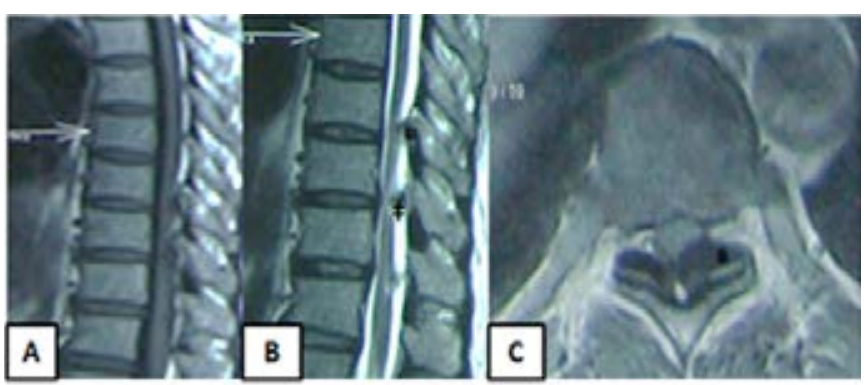

Figure $1 \mathrm{~A}$. T1 weighted MRI of thoracic spine showed continuous OLF between T9-11. B. T2 weighted MRI showed OLF (double dagger). C.Canal stenosis on Axial T2 weighted image due to OLF (asterisk)

Magnetic resonance imaging (MRI) (Fig.1) of thoracic spine showed posterior compression of the dural tube, in the area corresponding to the ligamentum flavum, with cord compression at Th 9-11 levels. MRI showed enlargement of the ligamentum flavum at the Th 9-11 level and stenosis in the thoracic canal. No discal pathology was reported, T1- and T2- weighted MR imaging disclosed a hypointense mass posteriorly and dorsal in the anatomical place of the ligamentum flavun. The patient was operated and the lamina of the Th 9-11 vertebra and the enlarge masses were removed en bloc. These masses were connected with the tissue of the ligament the Th-9 and Th-11 lamina, with no fixation of the bony masses, causing compression of the thoracic cord and caused myelopathy. Histological result as the ligamentum flavum approaches its laminal insertion, it has uncalcified and then calcified fibrocartilaginous features.

\section{Surgery}

Surgical decompression by laminectomy and resection of the hypertrophied ligaments must be made, supposed to be done as soon as possible after the onset of neurological symptoms and results can generally be improved Many techique of surgery can be performed decompressive laminectomy and excision of the ligamentum flavum, laminectomy combined with lateral fusion, en bloc laminectomy is suitable for the treatment of lateral-type and diffuse-type OLF, and the separating laminectomy is suitable for the thickened, nodular-type OL, decompressive laminectomy, laminoplasty and retaining posterior elements, fenestration, hemilaminectomy, and keyhole foraminotomy for the ossified lesions are advocated. In the present case, the patient underwent a posterior approach under general anesthesia in a prone position. A midline incision was made and the subperiosteal dissection until T8 to T12 lamina were exposed. Extensive drilling of the lamina T9 to T11 and then lamina removed, ossification of ligamentum flavum was drilling, and performed laminoplasty., ${ }^{4,92,13}$

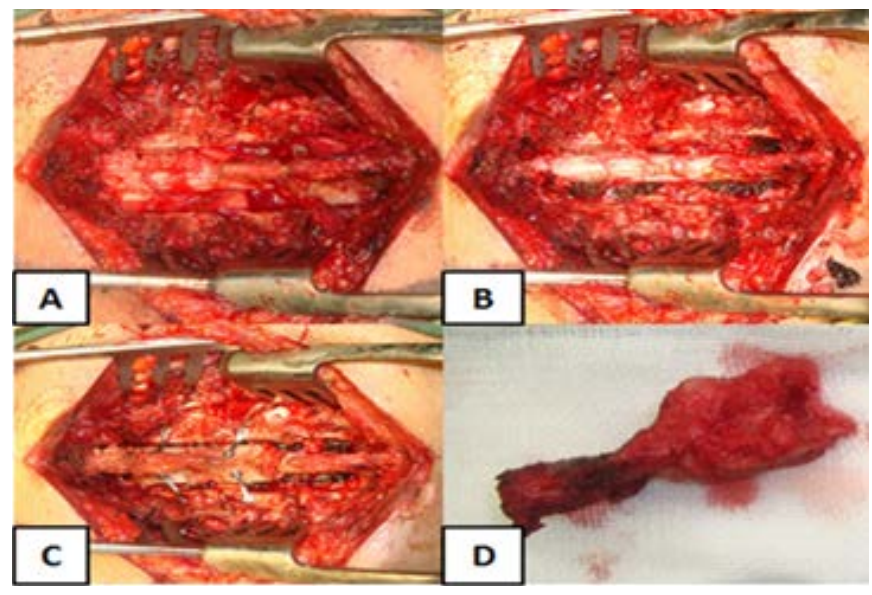

Figure 2 A After laminectomy, showed thickened and ossification of LF. B. Spinal cord decompressed after OLF was removed. C. Laminoplasty technique. D. Macroscopic OLF.

Histology specimen revealed that connective tissue fibres demonstrated penetrating the bone. The LF are made up chiefly of elastic connective tissue fibres longitudinally arranged with some thin bundles of collagen fibres and few spindle shaped fibroblasts. The elastic fibres are thinner than the collagen fibres. In the older age specimens, some fragmentation of elastic fibres was observed. Capillaries and very small thin walled blood vessels were not numerous and seen irregularly dispersed. No nerve trunks or endings were seen in the ligamentum flavum, osteochondral metaplasia and the presence of elastic fibers and chondrocytes (Fig 3) ${ }^{15}$. Patient underwent an intensive care unit stay for 24 hour, At hospital discharge day 6 patient remained paraparetic with a $3 / 3$ muscular strength, mild hypoesthesia in both lower limbs. At 1 year, he was able to walk with a cane, with no sphincter or sensory alterations.

\section{Discussion}

OLF is a pathological condition that causes neurological symptoms and usually occurs in the thoracic and less frequently in the cervical spine. It is more common in East Asian countries and usually affects adults 40 to 60 years of age. 
OLF is the most common compressive factor contributing to thoracic myelopathy in japanese, and was responsible for $60 \%$ of all thoracic cord compression either alone or combination with posterior longitudinal ligament ossification (OPLL) or in East Asia with an incidence rate between $2 \%$ to $4 \%$, Males is predominance $2.2: 1$, and the highest prevalence was in their sixties followed by fifties and seventies ${ }^{1,2}$. Ligamentum flavum has two portions, the interlaminar and the capsular. Ossification usually begins in the capsular portion and spreads to the laminar portion. The mechanism of hypertrophy and progression of ossification is confined to ligament flavum (LF) only and does not extend to the neighbouring spinal bony arch. The commonest surgical techniques of this are posterior decompression and laminoplasty both has been described as an alternatives due to late neurological deterioration and pain secondary to postlaminectomy kyphosis ${ }^{7-9}$.

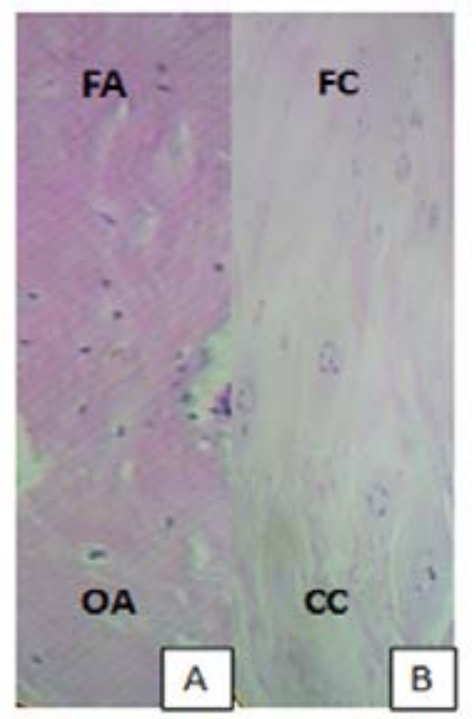

Figure 3 Histologic examination of the OLF. A. Section of the control ligamentum flavum showing a thin ossification front and regular arrangement of fiber bundles. B. From our patient the expanding ossification front, gathering many chondrocytes, and featuring an irregular of calcification.

FA: Fiber Area; OA: Ossification Area; FC: fibrocartilage; CC: Calcification Cartilage.

Ossification process starts at the base of the ligament with enchondral ossification of the vascularised fibrocartilaginous tissues. Studies have suggested cytokines as inflammatory marker, such as IL-6, TNF- $\alpha$, seem to play a crucial role in OLF, IL-4, IL-10, IL-16 and TGF-beta, contribute to the control of the magnitude to the inflammatory responses in vivo by the inhibition of the production of the pro-inflammatory cytokines or by counteracting many biological effects of proinflammatory mediators. Significant increases also in DNA synthesis and upregulated mRNA expression of types I, V, XI collagen and osteocalcin in LF cultures treated with various cytokines. LF cultures treated with IL-6, TNF-a, PGE2, and NO showed positive Von Kossa staining, indicating bone nodule formation from LF cells. There were also increased expression of TIMPs (Tissue Inhibitors of matrix metalloproteinase), especially TIMP-2, in ligamentum flavum fibroblasts result in hypertrophy of the ligamentum flavum in spinal stenosis by inhibiting MMP activity, but not by causing proliferation of ligamentum flavum fibroblasts or inhibiting apoptosis of ligamentum flavum fibroblasts. ${ }^{4,7,10}$

Numerous chondrocytes were present, especially around the calcification front, although no such chondrocytes were found in the normal ligamentum flavum suggested that the metaplastic chondrocytes in the ligamentum flavum are derived from mesenchymal cells or fibroblastlike cells, involvement of certain transcriptional factors in this process, Sox9, Runx2, Msx2 is known to promote chondrocyte differentiation of premature chondrocytes to hypertrophic chondrocytes and induces differentiation of premature mesenchymal cells and sometimes prevents the maturation of chondrocytes. In the normal ligamentum flavum, these factors might regulate chondrocyte differentiation, preserving homeostasis; however, overexpression of these factors in the OLF violates the regulation of chondrogenesis and differentiation of mesenchymal or fibroblast-like cells to mature chondrocytes in a complex autocrine/paracrine manner., ${ }^{3,4,16}$

The underlying etiology and pathogenesis of OLF are still unknown. It has been documented that the incidence of thoracic OLF is higher in patients with diffuse idiopathic skeletal hyperostosis, fluorosis, diabetes mellitus, ankylosing spondylitis (AS), and ossification of the posterior longitudinal ligament (OPLL), metabolic diseases such as Paget disease, hypoparathyroidism and X-linked hypophosphatemia. When conservative treatment is not effective, surgical treatment should be considered ${ }^{3,9}$ In native Indonesian $8.3 \%$ and chinese Indonesians found $62.2 \%$ found that HLA-B27+ an allele of the major histocompatibility complex shows a strong association carry a greater relative risk related spondiloarthropaties (SpA) or AS, Indians (Asian) 2-6\%, Japanese $<1 \%$ and Southeast Asians 5-12\%. ${ }^{17,18}$

\section{Conclussion}

Ossification of the ligamentum flavum has been widely recognized as a primary cause of thoracic myelopathy, even though the pathogenesis is still poorly understood. Mild symptoms should be conservative but if progressive neurologic deterioration surgery is mandatory. Further research and studies of OLF cases are necessary for early and proper diagnosis and therapy, in order to avoid a poor clinical outcome.

Declaration of interest: The authors report no conflicts of interest. The authors alone are responsible for the content and writing of the paper 


\section{References}

1. Aizawa T, Sato T, Tanaka Y, Ozawa H, Hoshikawa T. Thoracic myelopathy in Japan: epidemiological retrospective study in Miyagi Prefecture during 15 years. Tohoku J Exp Med 2006;210(3):199-208.

2. Yang Liu, Yongfei Zhao, Yu Chen, Guodong Shi, Wen Yuan. RUNX2 Polymorphisms Associated with OPLL and OLF in the Han Population. Clin Orthop Relat Res 2010;468(12):3331-41.

3. Uchida K, Yayama T, Cai HX, Nakajima H, Sugita D, Guerrero AR, et al. Ossification process involving the human thoracic ligamentum flavum: role of transcription factors. Arthritis Res Ther 2011;13(5):R144.

4. Ren L, Hu H, Sun X, Li F, Zhou JJ, Wang YM. The roles of inflammatory cytokines in the pathogenesis of ossification of ligamentum flavum. Am J Transl Res 2013;5(6):582-5.

5. Jaisuresh K, Vinodh Kumar P, Sundaravadivelu V. Thoracic myelopathy due to ossified hypertrophied ligamentum flavum. Calicut Med J 2007;5(5):e1.

6. Barnett GH, Hardy RW Jr, Little JR, Bay JW, Sypert GW. Thoracic spinal canal stenosis. J Neurosurg 1987;66(3):338-44.

7. Park JO, Lee BH, Kang YM, Kim TH, Yoon JY, Kim H, et al. Inflammatory cytokines induce fibrosis and ossification of human ligamentum flavum cells. J Spinal Disord Tech 2013;26(1):E6-E12.

8. Sanghvi AV, Chhabra HS, Mascarenhas AA, Mittal VK, Sangodimath GM. Thoracic myelopathy due to ossification of ligamentum flavum:a retrospective analysis of predictors of surgical outcome and factors affecting preoperative neurological status. Eur Spine J 2011;20(2):20515.
9. Yabe Y, Honda M, Hagiwara Y, Tohjo Y, Nakajima S, Ando A, et al. Thoracic radiculopathy caused by ossification of the ligamentum flavum. Ups $J$ Med Sci 2013;118(1):54-8.

10. Park JB, Lee JK, Park SJ, Riew KD. Hypertrophy of ligamentum flavum in lumbar spinal stenosis associated with increased proteinase inhibitor concentration. J Bone Joint Surg 2005;87(12):2750-7.

11. Brown JP, Lind RM, Burzesi AF, Kuo CK. Elastogenic protein expression of a highly elastic murine spinal ligament: the ligamentum flavum. PLOS One 2012;7(6):e38475.

12. Toledo JA, Isseldyk FV, Re M, Garrote M. Ossification of the ligamentum flavum as cause of thoracic cord compression: Case report of a Latin American man and review of the literature. Surg Neurol Int 2013;4:119.

13. Christiano LD AR, Goldstein IM. Ossification of the ligamentum flavum: a unique report of a Hispanic woman. Neurosurg Focus 2011;30:E15.

14. Muthukumar N. Dural ossification in ossification of the ligamentum flavum: a preliminary report. Spine 2009;34(24):2654-61.

15. Abdel-Meguid EM. An anatomical study of the human lumbar ligamentum flavum. Neurosciences 2008;13(1):11-6.

16. Kim HN, Min WK, Jeong JH, Kim SG, Kim JR, Kim SY, et al. Combination of Runx2 and BMP2 increases conversion of human ligamentum flavum cells into osteoblastic cells. BMB Rep 2011;44(7):446-51.

17. Nasution AR, Mardjuadi A, Suryadhana NG, Daud R, Muslichan S. Higher relative risk of spondyloarthropathies among B27 positive Indonesian Chinese than native Indonesians. J Rheumatol 1993;20(6):988-90.

18. Khan MA. Thoughts concerning the early diagnosis of ankylosing spondylitis and related diseases. Clin Exp Rheumatol 2002; 20(6 Suppl 28):S6-S10. 